\title{
NLM's Library Network: A Force for Outreach
}

\author{
Jean P. SHIPMAN M.S.L.S. ${ }^{\mathrm{a}, 1}$, Catherine M. BURROUGHS M.L.S. ${ }^{\mathrm{b}}$ \\ and Neil RAMBO M.L. ${ }^{\mathrm{c}}$ \\ ${ }^{a}$ Librarian Emerita, Spencer S. Eccles Health Sciences Library, University of Utah, U.S.A. \\ ${ }^{\mathrm{b}}$ Health Sciences Library, University of Washington, U.S.A. \\ ${ }^{\mathrm{c}}$ Grossman School of Medicine, New York University, U.S.A.
}

\begin{abstract}
This chapter considers the transformation of U.S. National Library of Medicine's (NLM) national network of libraries into an effective force for spreading awareness of NLM's resources, services, and tools and increasing their use. Several examples of network programs and projects are recounted to illustrate the influence of NLM's longest serving Director, Donald A.B. Lindberg M.D. on the development and evolution of NLM's library network.
\end{abstract}

Keywords. Outreach, National Network of Libraries of Medicine (NN/LM0, Regional Medical Library, U.S. National Library of Medicine, Donald A.B. Lindberg M.D.

\section{Introduction}

The Medical Library Assistance Act of 1965 did several things to improve medical library facilities, resources, automation of library services, and training of librarians. The Act also led to the creation of the Regional Medical Library (RML) network, coordinated by the U.S. National Library of Medicine (NLM), which was designed to facilitate resource sharing among hospital libraries, academic libraries, and NLM. This network was successful in improving access to medical information for health professionals connected to its nodes. By the $1980 \mathrm{~s}$, it became increasingly clear many health professionals did not have access to a medical library nor access to the latest medical information. The development in personal computer technology and public communication networks began to make it possible for individuals to get access to information without having physical access to a library.

Appreciation of these developments led Congress, in 1987 and again in 1988, to encourage NLM to develop an outreach program to reach health professionals in all areas, including rural and other under-served areas, and to amend NLM's mandate to include “...publicize the availability of [its] products and services..." [1]. In response, the NLM Board of Regents commissioned an outreach planning panel in 1988, chaired by Dr. Michael E. DeBakey. The panel delivered a report to the Board entitled Improving Health Professionals' Access to Information [2]. The Board approved the report in 1989.

\footnotetext{
${ }^{1}$ Jean P. Shipman, 311 Cedar Meadow Drive, Nellysford, VA 22958 USA; E-mail: jean.shipman@utah.edu
} 
Among the key recommendations of this influential and far-reaching report was the refashioning and retooling of the RML network into a national "field force" for NLM. Other recommendations addressed new and expanded grant programs, training programs in biomedical information management, and development of new products and services. Results of these developments are covered in other chapters in this book. This chapter focuses on the National Network of Libraries of Medicine (NN/LM), renamed as recommended in the report to emphasize its national structure and direction [See Figure 1].

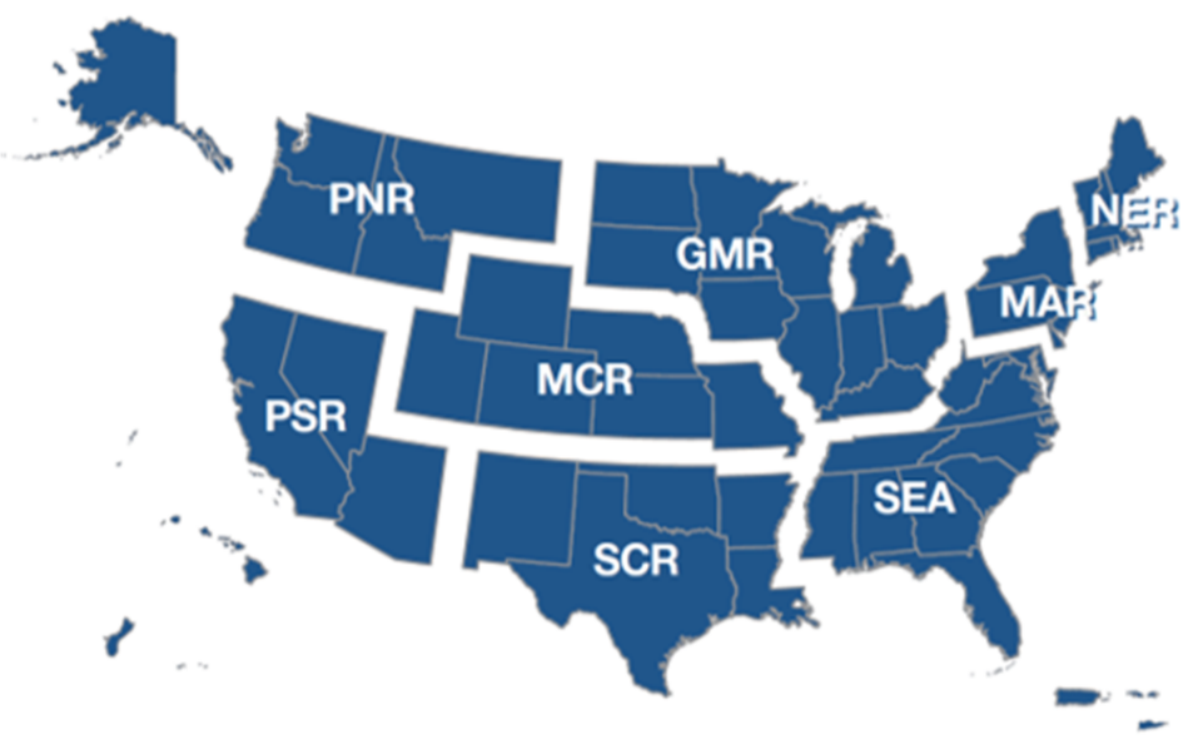

Figure 1. NN/LM Map - showing the Regional Configuration from 1991 to April 2021

None of this would have happened without the vision and efforts of Donald A.B. Lindberg, M.D., Director, U.S. NLM. He arrived at the Library in 1984 with great admiration for the Network and a desire to expand its scope and increase its effect.

Wallingford et. al. provide a detailed review of the first five years of NLM's outreach programs (1990-95), including programs performed by the NN/LM [3]. This chapter focuses on key NN/LM outreach projects and initiatives following this initial period. The areas highlighted are necessarily selective of the whole and represent Dr. Lindberg's particular interests and influence. Speaker's history of the NN/LM from 1985-2015 gives a broader overview of the program during Dr. Lindberg's tenure as NLM Director [4].

\section{Initiatives}

\subsection{Information Access}

With his FY2013 statement serving as an exemplar, in each annual Opening Statement to the House Subcommittee on U.S. Labor-HHS-Education Appropriations, Dr. 
Lindberg advocated strongly for NN/LM's role as NLM's outreach program: “To be of greatest use to the widest audience, NLM's information services must be known and readily accessible" [5]. NN/LM was indeed a major force in raising awareness and increasing effective use of NLM services, databases, and tools. For example, through $\mathrm{NN} / \mathrm{LM}$ resource sharing agreements and training by dedicated NN/LM staff, librarians established coordinated interlibrary loan and document delivery services using NLM's resource sharing products and systems. These included DOCLINE for interlibrary lending and borrowing, LinkOut to lead PubMed users to their libraries' collections, and Loansome Doc linked to Grateful Med, and later PubMed, to provide users the ability to request journal articles at the click of a button. Through delivery of documents--digital images or copies, and interlibrary lending of books and audiovisuals - NN/LM's resource sharing program offers health professionals access to resources not limited to a single local library. Time is nearly always of the essence in document delivery and interlibrary loan of health information. A smoothly functioning resource sharing program minimizes the time lapse between requesting an article, book, or audiovisual and its receipt.

\subsubsection{Loansome Doc}

Dr. Lindberg wanted anyone searching for journal article citations and abstracts through Grateful Med and later PubMed to easily obtain the retrieved articles' full-text information, including those unaffiliated with a health sciences library. Thus, the advent of Loansome Doc. This feature encouraged users to register with an NN/LM library to obtain physical copies of journal articles through clicking on article citations identified in their literature searches. The user established a profile with a library and agreed to its service terms and prices. Library details were added to the user's Loansome Doc profile to create the link between the user and the library.

Loansome Doc was tested by the Pacific Southwest RML of the NN/LM in 1990-91. NN/LM regional offices conducted extensive training for health sciences librarians to familiarize them with the system and to understand what policies were needed to deliver journal content. NN/LM also exhibited the service and performed associated training sessions at health professional conferences.

Initially, there was resistance to Loansome Doc from some librarians, mostly because unaffiliated users were a new audience for them to serve. They had to decide who they would serve, if and how they would bill for the service, and how they would collect associated fees. Dr. Lindberg spoke at several librarian conferences to hear their concerns and to share his reasoning for wanting such a valuable content delivery service. His interactions with rural physicians during his years at the University of Missouri, Columbia provided firsthand insight about the need and usefulness of Loansome Doc.

\subsubsection{Internet-based Access}

Under Dr. Lindberg's leadership, NLM modernized other resources and tools to "fundamentally [change] the way biomedical knowledge and health information is collected, organized, and made available for public use" [6]. Health providers and scientists gained new or improved access to medical literature via PubMed and PubMed Central and to clinical trials and their results via ClinicalTrials.gov. The general public became a significant NLM user group once NLM's databases became freely available via the Internet. In 1998, NLM launched MedlinePlus, followed by MedlinePlus en español in 2002. These companion Internet-based resources for patients and their 
families offer free and up-to-date information about diseases, conditions, and wellness issues in understandable language.

To inform user groups about these resources, NN/LM sponsored or conducted hundreds of exhibits annually at state, regional, and national professional and scientific meetings for health professionals, researchers, and librarians. The exhibits increased NN/LM's visibility while identifying NLM as a leader in biomedical information technology research and a developer of information systems [7]. RMLs also embarked on initiatives to add public libraries and community organizations to NN/LM and to train these groups about NLM Internet resources as authoritative sites for the public. By 2015, more than 6,000 academic health sciences libraries, hospital libraries, public libraries, and community-based organizations had joined NN/LM in its mission to bring highquality information to health professionals and the public--regardless of location, socioeconomic status, or access to computers and telecommunications, putting NN/LM in a solid position to "bring the message about NLM's free, high-quality health information resources to communities across the nation" [5].

\subsection{Community-based/Underrepresented Populations Collaborations}

Engaging communities, especially minority and underserved populations, to learn more about their personal health through collaboration with and training by librarians was a shared value of Dr. Lindberg and NN/LM. Many NN/LM outreach initiatives focused on specific populations, such as unaffiliated health professionals (e.g., public health and rural health care professionals), community-based organizations, underserved minority communities, and non-medical librarians. With NLM funding, health science librarians partnered with communities to assess their information needs, learn about their political structures and health beliefs, and conduct - in concert with the communities - activities targeted to address the identified needs.

\subsubsection{Public Health Partnerships}

At its core, the initial target audience of NN/LM outreach to underserved and rural health professionals was physicians who did not have access to a medical library. The information needs of physicians were better understood than for most other health professionals. Access to the clinical literature through NLM tools and services was well suited to meet many of those needs. There was a good alignment between the needs, services, and tools, and audience. During the 1990s, outreach to other segments of underserved health professionals increased, e.g., the public health workforce - at the local level, as did awareness that the information needs of these diverse groups were less well understood.

The Partners in Information Access for the Public Health Workforce (Partners), a multifaceted collaboration, had its origin in a 1994 request to Dr. Lindberg from the Assistant Secretary for Health, Department of Health and Human Services, for help in improving public health access to and use of the emerging National Information Infrastructure. Formation of the partnership was recommended in an influential report from a meeting held at NLM in 1995. The meeting and the report reflected a shared sense that more was needed to adequately serve the diverse needs of public health professionals [8]. Several government agencies and professional organizations and associations came together under the auspices of NLM and the U.S. Center for Disease Control (CDC) to share information and resources. The Partners compiled resource guides and provided 
training in finding and using information resources to public health staff to support informed practice.

The Partners enabled public health entities to learn about the resources and services of NLM and to make them known to their members and constituents. Also, many librarians were trained through the Partners in the diverse and complicated information needs of the public health workforce. This collaboration was at the core of what made the Partners successful.

\subsubsection{Public Health Department Connections}

Parallel with the development of the Partners, in the late 1990s, the potential of connecting computer networks for communication and information access was beginning to be realized. One example of this at the local level was a project to connect local health departments in Washington State to the state health department and the Internet. The project officers saw this as an opportunity to piggyback on a technology project and use it to train public health staff to more readily incorporate access to information, data, health guidelines, and other resources. The CDC and NN/LM were brought together through the Northwest Center for Public Health Practice, based at the University of Washington (UW), to coordinate and offer training at local health departments, once technological connections were established. The training sessions also provided an opportunity to conduct information needs assessments of segments of the public health workforce at the local level [9].

In 2010, the New England Region (NER) of NN/LM received funding to create a digital library tailored to public health. Seven public health departments were provided free digital access to licensed electronic resources. The supporting librarian team learned Internet access, hardware availability, online searching training, free full-text access to licensed resources, and support from upper leadership were all needed to facilitate evidence-based decision making by public health workers [10].

The NER-sponsored public health information access digital library project led to the creation of a customized digital library of no-cost full-text journal articles and books for state public health departments, first in New England and the state of Colorado. In addition to access to evidence-based resources, online searching classes, and searching consultation services were provided. An interlibrary-loan network was also established to obtain items not provided by the digital library. The digital library grew in type and quantity of full-text resources and databases and expanded its reach to other state public health departments.

\subsubsection{MedlinePlus Go Local}

At the 2010 Annual Meeting of the U.S. Medical Library Association (MLA) in Washington, D.C., U.S., a bittersweet celebratory gathering, including Dr. Lindberg, was held to sunset the Go Local projects funded by the NLM [11]. During the ten-year period of Go Local, more than 30 libraries and library consortia had developed Go Local websites.

Go Local was created by NLM in 2001 as a vehicle for linking users of the MedlinePlus consumer health information site to and from local quality health service and locator information. The project, overseen by RML programs across the country, provided libraries and library consortia with start-up funds for outreach to communitybased organizations and local health agencies. The project provided "one-stop shopping" for access to nationally applicable information from MedlinePlus connected to and from 
essential state and local health and human services-related resources. This project advanced connections with communities, public health departments, and other agencies supporting health information needs. The Go Local projects also created a community of practice among the Go Local sites. Site personnel met regularly to hear updates and share engagement strategies.

The Southeastern Atlantic (SEA) Region of NN/LM was a hotbed of Go Local sites, with seven Go Local projects funded, including the original prototype, NC Health Info. Each project reflected the unique characteristics of its state or home institution. For example, the Health Sciences and Human Services Library at the University of Maryland-Baltimore focused on assessing community health information needs, and then building relationships with community organizations and information providers across the state. The site was launched with a gala ribbon-cutting ceremony attended by local politicians, community members, NLM staff, and public health officials. Then Baltimore Health Commissioner, Dr. Joshua Sharfstein, thanked the Maryland Health Go Local team on behalf of the beneficiary citizens of Maryland.

The expense of sustaining the Go Local projects, and the growth of access to rapidly updated local health service information via Internet search engines and Internet-based health information sites, impacted the use and future need for this program. The Go Local websites served as models for other health information websites. There were many lessons learned about partnering with community-based organizations and with talented colleagues at NLM and across the country on a project. Many participants consider Go Local the most worthwhile project in which they have been involved. NLM's decision to cease support for Go Local is an example of Dr. Lindberg's willingness to let data about usage, sustainability, competition, and cost overrule his personal preferences.

\subsubsection{Information Prescriptions (Rx)}

Dr. Lindberg's understanding of the power of doctor-patient trusted relationships led him to encourage the use of information prescriptions. As he knew first-hand, many patients respond to a medical order from their provider. Using this same premise, if a provider issued a written order for information, Dr. Lindberg felt patients would be more likely to go to a library or online to obtain health information related to their conditions.

The NLM Information Rx initiative was officially launched in partnership with the American College of Physicians in New Orleans, Louisiana, on April 22, 2004 [12]. Dr. Lindberg introduced the concept and encouraged his medical colleagues to embrace it and refer patients to quality information, written for the lay person and delivered online via NLM's MedlinePlus. NLM created "prescription" pads with the MedlinePlus URL and space to notate the condition to be researched. The pads were small enough to fit into provider pockets for easy access.

Promotion of the Information Rx initiative was performed by NN/LM. Each region publicized the no-cost availability of the Rx pads to its members. Network librarians informed providers within their institutions and assured them they would be willing to assist patients with filling the information prescriptions. Professional library associations spread the word about the Information Rx pads so patients going to public libraries would be assisted by librarians familiar with the concept.

The Tompkins-McCaw Library at Virginia Commonwealth University received special NLM funding to identify barriers related to issuing information prescriptions at its clinical Women's Health Center located in Richmond, Virginia. Center providers were educated about the initiative and were eager to participate. Despite the initial 
eagerness, time constraints with patient visits often did not provide the chance to explain the concept and issue the prescriptions, or providers simply forgot to do so [13]. These factors were common barriers. When not a significant issue in the many settings where the project was successfully deployed, Information Rx proved Dr. Lindberg correct in his belief that providers can influence patient behavior.

After several years, NLM phased out the printed Rx pads in favor of experimenting with a protocol for direct connection from electronic health records. This service, called MedlinePlus Connect, is still in heavy use today. Several librarians partnered with their electronic health record departments to add quality health information to patients' personal health records and to create an automated way for providers to issue health information via patient visit summaries. The concept of provider referrals influencing patient information-seeking behavior, highlighted by Dr. Lindberg's Information Rx efforts, proved to be a long-term success.

\subsubsection{Symposium on Community-based Health Information Outreach}

An example of Dr. Lindberg's commitment to programs designed to improve the quality of life and eliminate health disparities is NLM's support for the 2004 Symposium on Community-based Health Information Outreach, proposed by the University of Utah and the New York Academy of Medicine. The Symposium encouraged participants to increase their awareness of barriers to accessing reliable health information and to consider potential innovative solutions designed to improve information access, especially for traditionally marginalized populations [14].

The Symposium was held at NLM's Lister Hill Center on December 2 and 3, 2004, and was streamed live to a worldwide audience. The two-day event included 150 participants from various backgrounds and experiences, including librarians, community-based workers, and evaluation specialists. The Symposium's primary goals were to discuss the use of information and communication technologies to develop new community engagement models and to demonstrate how using these new technologies could increase the capacity of health sciences libraries to deliver programs and services beyond their traditional boundaries. The Symposium also was an opportunity to explore new ideas in consumer health information outreach, to consider increasing access to health information through community-based organizations, and to discuss NLM's current and future efforts to reduce health disparities for all underrepresented communities, with a particular focus on American Indian/Alaska Native populations.

The Symposium planning committee consisted of representatives from NLM and representatives from several RMLs. The program included keynote speakers, papers, and posters. Presenters focused their discussions on the importance and impact of NLM's outreach, methods for assessing complex outreach activities, strategies librarians can deploy when engaging with community-based organizations, and recommendations on next steps for supporting outreach to underrepresented communities.

The Symposium concluded with a consensus NLM and NN/LM should continue their outreach to underrepresented and traditionally marginalized communities and to work toward developing partnerships and collaborations with community-based organizations, as key strategies to extend the reach of NLM and NN/LM. Participants encouraged NLM and NN/LM to continue investing in the design and implementation of projects considering complex cultural structures, as well as recognizing the deep-seated challenges faced by communities with the most need [15]. 


\subsubsection{American Indians/Alaska Natives}

As a result of a 1995 review of NLM's outreach programs, NLM determined more attention was required to engage American Indian/Alaska Native communities. Subsequently, NLM funded several important programs, including the Tribal Connections projects, and NLM's exhibition "Native Voices" about Native beliefs and practices regarding health and wellness. Dr. Lindberg's influence and vision was central to increasing NN/LM outreach to American Indians/Alaska Natives.

\subsubsection{Tribal Connections}

NN/LM's health information outreach initiative, known as Tribal Connections (TC), was a constellation of collaborations among American Indian/Alaska Native communities and health science libraries. The TC program was initially designed in three phases - TC I, II, and III - which ran between 1998 and 2003. These first three phases were implemented by the NN/LM Pacific Northwest Region RML (PNR), located at the University of Washington's Health Sciences Library, with funding and partnership support from NLM. The goal of TC I was to improve access to the Internet for 16 Alaska Native/American Indian communities in the northwest United States. PNR worked with each community to identify what was needed to improve access and use of health information. The tribes emphasized Internet connectivity as their number one need. Without reliable connections, the growing numbers of authoritative Internet resources were simply out of reach. With PNR funding, each community developed local strategies for improving connectivity through a community-based approach to project planning and implementation [16]. Community members participated in NN/LM training on digital health literacy and consulted with NN/LM about culturally relevant health information. TC II focused on American Indian communities in the southwest. TC III implemented community-based approaches to increasing the use of reliable health information through training and education in the states of Idaho, Oregon, and Washington.

In 2001, TC IV, also known as Tribal Connections Four Corners (TC4C), was created with heavy influence from the initial TC program, as well as the Tribal Health Connections Project, funded by the Bill \& Melinda Gates Foundation. At the time, the TC4C project was the largest collaborative outreach project designed to engage American Indian communities in NLM's history and included the involvement of three NN/LM regional offices, the MidContinental Regional Medical Library (MCR) at the University of Utah, South Central Regional Medical Library (SCR) at the Houston Academy of Medicine--Texas Medical Center, and the Pacific Southwest Regional Medical Library (PSR) at University of California, Los Angeles. Four Resource Libraries joined in the effort, including the University of Arizona, University of New Mexico, University of Colorado, and University of Utah. TC4C made funding available to organizations, including public libraries serving American Indian populations, and it offered training on the use of NLM resources.

Two important features of the TC projects were increased community engagement and infrastructure improvements. Early in the project, staff assessed the technology needs of the participating tribes, which resulted in improving access to the Internet through upgrades to computers and connectivity. The project also increased the number of partnerships between tribes and organizations interested in sharing technology and infrastructure resources. Another important achievement was funding dedicated to tribal community outreach coordinators and professional personnel who worked to implement the project's goal of increasing access to health information to participating communities. 
These coordinators focused on developing connections, trust, and familiarity to improve their encounters with tribal communities. The combination of these areas of outreach and technology resulted in an increase in community members' proficiency in the use of computers and interest in using the Internet to find NLM resources to answer healthrelated questions. The TC project also deepened NN/LM's understanding of culturally appropriate health information outreach with American Indian/Alaska Native communities [17].

\subsubsection{Native Voices Healing Totem}

In September 2011, the University of Washington Libraries and the Native American Land Conservancy celebrated the blessing of a beautiful healing totem commissioned by NLM in honor of the new NLM exhibition focusing on Native views and definitions of health and illness. Lummi Indian master carver Jewell Praying Wolf James, a worldrenowned master carver of totems or healing poles, crafted NLM's healing totem. Mr. James is the lineal nephew of Chief Seattle (for whom the city was named), and the head carver for the House of Tears Carvers of the Lummi Indian Nation in Bellingham, Washington. The totem blessing and celebration, held at the Seattle Center on September 11, 2011, launched a series of totem blessings attended by NN/LM representatives in cities and tribal communities across the country, as the totem traveled to the East coast, with final placement in front of NLM on the National Institutes of Health campus. In its permanent home, the totem became a dramatic focal point of the NLM exhibition, Native Voices: Native Peoples' Concepts of Health and Illness.

In 2011, the University of Pittsburgh Health Sciences Library System became the RML for the states of Delaware, New Jersey, New York, and Pennsylvania. In starting a new RML, Renae Barger, Executive Director, was recruiting staff when asked by NLM to attend blessings and write blog postings at two stops on the NLM Healing Totem's journey to tribal sites in New York. Coincidently, incoming Outreach Coordinator, Kate Flewelling, was preparing to move to Pittsburgh from Syracuse, N.Y., minutes from the Onondaga Nation Reservation, one of the journey stops. Even before her official start date, Flewelling had her first outreach assignment. She witnessed the Opening Ritual, which included a regular meeting of the Chiefs' Council in the Nation's language and an introduction to the Totem from Jewell Praying Wolf James. As a new outreach staff member, Flewelling learned the importance of listening as a key component of outreach and community engagement.

A few days later, Barger attended a two-day ceremony at Arrow Park, N.Y., the home of another Healing Totem, dedicated by the same master carver in honor of the victims of September 11. In 2002, the Lummi Indians dedicated the Healing Totem and marked Arrow Park as a special place of healing. Since then, Arrow Park has been a site for an annual tree planting to honor $9 / 11$ victims. It is a dedicated site of professional training programs about bereavement, suicide prevention, and post-traumatic stress disorder. Barger remembers learning about NLM's Healing Totem's journey and the meaning behind its markings. The sense of respect and pride, and the overwhelming appreciation of Dr. Lindberg for driving this important effort to showcase and preserve cultural traditions of healing practices were evident. 


\subsection{Influence on NN/LM Services}

Dr. Lindberg long recognized the leadership role of RMLs in guiding changes and improvements to NN/LM services. In this section, three health science libraries, serving as RMLs during Dr. Lindberg's tenure, describe important initiatives NLM supported enabling RMLs to: 1) build capacity for evaluation by NN/LM network members and RMLs; 2) use NN/LM evaluation tools and resources to pilot and communicate the impact and results of a new decentralized RML; and 3) foster the role of librarians in eScience.

\subsubsection{Support for NN/LM Evaluation}

In the mid-1990s, outreach to underserved populations became one of Dr. Lindberg's highest priorities for NN/LM. Acknowledging many NN/LM librarians sought guidance about ways to evaluate their outreach programs, NLM conceived, funded, and oversaw a specialized outreach planning and evaluation study.

NLM's Office of Health Information Programs Development division provided leadership for the study, conducted by the PNR. A group of 18 national experts advised the evaluation study, to add their multi-disciplinary perspectives to library outreach evaluation practices. White papers authored by several of the advisors provided a theorybased framework for the resulting NN/LM evaluation guide: Measuring the difference: guide to planning and evaluating health information outreach [18]. This 130-page guide, first published in 2000, is still considered a primary evaluation resource of the NN/LM. About 4,000 copies have been distributed to U.S. and international organizations, such as libraries in hospitals, medical centers, and universities, as well as departments of public health, faith-based organizations, and all kinds of non-medical libraries.

Between 2001-2015, NLM funded and supported the Outreach Evaluation Resource Center (OERC), based at the PNR, to help NN/LM members and RMLs collect, understand, and act on information about their projects to plan and improve their programs and adapt them to changing environments. OERC developed and published a booklet series to supplement NN/LM's evaluation guide [19-21]. The booklet series simplified the evaluation process described in the guide, to make it less overwhelming to anyone with minimal background in research and evaluation. The booklet series was last updated in 2013, and it is available in print or online at no cost. Roughly 3,000 print copies have been distributed globally. NLM continued to fund a National Evaluation Office at the PNR until 2021.

\subsubsection{A Decentralized Regional Medical Library}

The University of Utah's Spencer S. Eccles Health Sciences Library's (EHSL) proposal for the MidContinental Region (MCR) of NN/LM was accepted in 2001; it focused on a technology-based, decentralized implementation of resources, including staff. Unlike most other Regions in the NN/LM, each state in the Region had only one Resource Library, which hired a local librarian to get to know the people, communities, and organizations in their state to match appropriate NLM resources with their unique health information needs. This resulted in greater breadth and scope of outreach activities, deeper relationships with partners within each state, and a significant pool of individuals with whom to engage in project development and funding applications. Prior, all RMLs had a centralized operations office. The EHSL proposal distributed the technology and 
the money to the state Resource Libraries, thus providing them with an immediate benefit of MCR participation and ensuring their work commitment.

Discussions of the distributed model among the Resource Library Directors (RDLs) began as early as 1993, while the RML functioned in the typical centralized model at the University of Nebraska. All RDLs were keenly interested in the potential for working together in a new way, utilizing new technologies to support communications and collaboration. Dr. Lindberg was enthusiastic about the ideas presented in University of Utah's proposal for two reasons. First, this new model was dependent on applying technology to aid communication, planning, and outreach to each state. Second, he was convinced a model based upon greater collaboration among key libraries would better forward NLM's goal to bring reliable health information to more people.

Regular meetings and communication with RDLs and their MCR librarian staff allowed for strategic program planning, collaboration, and program evaluation resulting in clear expectations, goals, and achievements. The experience with program evaluation fed into the growing movement among librarians to use data for decision making, demonstrating their value to administrators, and improving their research skills. The "Measuring Your Impact" workshop developed by staff at MCR and PNR taught hundreds of librarians to collect data to evaluate their resources and services. A website was developed to collect financial data and provide means to measure cost-benefitanalysis and return on investment [22].

\subsection{3. eScience}

Spurred by NLM's advances in bioinformatics and policies for open science to drive scientific discovery, NN/LM embarked on new initiatives, such as eScience programs, promoting the librarians' role in research data management. NER developed the first eScience program.

Recognizing the need to address eScience as a field of practice intersecting with diverse constituent groups such as library specialists, IT specialists, and researchers, NER sponsored the first eScience Symposium in 2009. It convened librarians and information scientists from basic sciences, health sciences, medical centers, and general academic libraries to explore the various roles eScience could entail for the community. Based on feedback and enthusiasm displayed by attendees, work began to assess needs and develop resources to enhance basic science knowledge of librarians, while also gathering resources focusing on the principles of eScience best practices. An assessment of biomedical and science librarian eScience learner and user needs was conducted to compile and organize an online portal assembling the tools needed to address eScience capacity building. Working with key participants in NER, advisory and editorial boards were established, and the eScience Portal for New England Librarians was born [23].

Subsequent symposia, professional development programs, and extended eScience Boot Camps were established to continue capacity building, expand data literacy, and advance partnerships and collaborations. Through these programs, NER became the academic home for New England area librarians and consortia partners with a shared interest in fostering the role of librarians in data science research initiatives.

NER eScience efforts served as a model for addressing the research data management needs of librarians and researchers in other NN/LM regions [24]. Through NLM's invaluable funding support, NER's development and implementation of its eScience program and community of interest equipped network members with a new understanding of eScience and the roles librarians play in helping their researchers 
manage, preserve, curate, and share data. By 2010, eScience initiatives were a required program element in NLM's Request for Proposals for RML programs in 2011-2016.

\section{Summary}

The depth and breadth of Dr, Lindberg's influence upon NN/LM is hard to quantify, as he was either directly or indirectly promoting access to the medical literature and libraries to health care providers and the public to improve the nation's health. This chapter offers some key highlights of his extensive involvement and guidance. It is by no means an allinclusive collection. There are many more NN/LM outreach activities and efforts not reflected in this chapter. Readers are encouraged to consult the various journal articles listed in the references to learn about others. In addition, NN/LM did not perform outreach initiatives in isolation, but often partnered with NLM's Specialized Information Services Division, Office of Health Information Programs Development, and National Information Center on Health Services Research and Health Care Technology. Through such internal collaborations, more citizens benefited from NLM's outreach activities under Dr. Lindberg's expansive and informed direction.

\section{Acknowledgements. Contributing Authors}

The chapter authors wish to thank the following individuals for contributing to the specified sections of this chapter. These individuals were all part of the NN/LM--their specific RML host institutions noted--when the described projects were conducted.

University of California, Los Angeles - Julie Kwan (2.1.1); University of Maryland, Baltimore -Tony Nguyen (2.2.3), Jean Shipman (2.1.1, 2.2.4), Mary Joan (M.J.) Tooey (2.2.3); University of Massachusetts Medical School, Worcester - Elaine Martin, DA (2.2.2, 2.3.3); University of Pittsburgh -Renae Barger (2.2.6.2), Kate Flewelling (2.2.6.2); University of Utah - John Bramble (2.2.5, 2.2.6.1), Claire Hamasu (2.2.5, 2.2.6.1, 2.3.2), Wayne Peay (2.3.2), Gerald Perry (2.2.6.1); Catherine Soehner (2.2.5, 2.2.6.1, 2.3.2), Deborah Ward (2.3.2); University of Washington -Tania Bardyn (2.2.5, 2.2.6.1, 2.2.6.2, 2.3.1), Catherine Burroughs (2.2.5, 2.2.6.1, 2.2.6.2, 2.3.1), Neil Rambo (2.2.1, 2.2.2).

\section{References}

[1] U.S., Congress, Joint resolution amending the National Library of Medicine Act, Public Law 100-202, section $215,1987$.

[2] National Library of Medicine (U.S.). Outreach planning panel. improving health professionals' access to information: challenges and opportunities for the National Library of Medicine. 1989 May [cited 2021 May 3]; Available from: https://collections.nlm.nih.gov/catalog/nlm:nlmuid-101627944-bk

[3] Wallingford KT, Ruffin AB, Ginter KA, Spann ML, Johnson FE, Dutcher GA, Mehnert R, Nash DL, Bridgers JW, Lyon BJ, Siegel ER, Roderer NK. Outreach activities of the National Library of Medicine: a five-year review. Bull Med Libr Assoc. 1996 Apr;84(2 Suppl):1-60.

[4] Speaker SL. An historical overview of the National Network of Libraries of Medicine, 1985-2015. J Med Libr Assoc. 2018. April;106(2):162-74. DOI: 10.5195/jmla.2018.297.

[5] Lindberg, DAB. NLM opening statement FY2013. NLM congressional justifications, statement for the Record House Subcommittee on Labor-HHS-Education Appropriations. [Internet] U.S. National Library of Medicine; 2012 [cited $2021 \mathrm{Feb} 15$ ]. Available from: https://www.nlm.nih.gov/about/2013openst.html

[6] MacKay T. NLM Board of Regents approves resolution honoring NLM Director Lindberg [Internet]. 2015 Feb [cited 2021 May 3] p. 1. Available from: https://wayback.archive-it.org/org350/20180911191348/https://www.nlm.nih.gov/news/bor_resolution_lindberg_2015.html 
[7] Hajarian K, Ruffin A, Charuhas J, Yancey T, Carr A, Champ-Blackwell S, Editors. National Library of Medicine exhibit management manual. 3rd ed. Bethesda, MD: National Library of Medicine; 2005.

[8] Cahn MA, Auston I, Selden CR, Cogdill K, Baker S, Cavanaugh D, Elliott S, Foster AJ, Leep CJ, Perez DJ, Pomietto BR. The Partners in Information Access for the Public Health Workforce: a collaboration to improve and protect the public's health, 1995-2006. J Med Libr Assoc. 2007 Jul;95(3):301-9. DOI: 10.3163/1536-5050.95.3.301.

[9] Rambo N, Dunham P. Information needs and uses of the public health workforce--Washington (State), 1997-1998. MMWR Weekly. 2000 Feb 18;49(06):118-20.

[10] LaPelle NR, Dahlen K, Gabella BA, Juhl AL, Martin E. Overcoming inertia: increasing public health departments' access to evidence-based information and promoting usage to inform practice. Am J Public Health. 2014 Jan;104(1):77-80.

[11] Klein LJ. NLM to discontinue support of MedlinePlus Go Local. NLM Tech Bull. 2010 Mar-Apr; 373. [Internet] [cited 2021 June 28] https://www.nlm.nih.gov/pubs/techbull/ma10/ma10 go local discontinue.html

[12] National Library of Medicine (U.S.). National Library of Medicine programs and services FY2004. Bethesda, MD: The Library; 2004 p. 1.

[13] Leisey MR, Shipman JP. Information prescriptions: a barrier to fulfillment. J Med Libr Assoc. 2007 Oct;95(4):435-8.

[14] Peay WJ, Rockoff ML. Proceedings of the symposium on community-based health information outreach, December 2-2, 2004, Bethesda, Maryland, USA. J Med Libr Assoc. 2005 Oct;93(4 Suppl):S4-92.

[15] Peay WJ, Rockoff ML. The National Library of Medicine's 2004 "symposium on community-based health information outreach”. Introduction. J Med Libr Assoc. 2005 Oct;93(4 Suppl):S4-9.

[16] Press N, Sahali R, Burroughs C, Frank K, Rambo N, Wood FB, Siegel ER, Fuller SS. Program management and policy issues in information outreach. J Health Soc Policy. 2003 Sep 1;17(3):1-20.

[17] Wood FB, Sahali R, Press N, Burroughs C, Mala TA, Siegel ER, Rambo N, Fuller SS. Tribal connections health information outreach: results, evaluation, and challenges. J Med Libr Assoc. 2003 Jan;91(1):57-66.

[18] Burroughs, CM, Wood FB. Measuring the difference: guide to planning and evaluating health information outreach - Digital Collections - National Library of Medicine [Internet]. Seattle, Wash: National Network of Libraries of Medicine, Pacific Northwest Region; Bethesda, MD: National Library of Medicine. 2000. [cited 2021 May 9]. Available from: http://resource.nlm.nih.gov/100961143

[19] Olney CA. Getting started with community-based outreach - Digital Collections - National Library of Medicine [Internet]. Seattle, Wash: National Network of Libraries of Medicine, Pacific Northwest Region; Bethesda, MD: National Library of Medicine. [cited 2021 May 9]. Available from: http://resource.nlm.nih.gov/101276145

[20] Olney CA. Including evaluation in outreach project planning - digital collections - National Library of Medicine [Internet]. Seattle, Wash: National Network of Libraries of Medicine, Pacific Northwest Region; Bethesda, MD: National Library of Medicine. 2006. [cited 2021 May 9]. Available from: http://resource.nlm.nih.gov/101276146

[21] Olney CA. Collecting and analyzing evaluation data - digital collections - National Library of Medicine [Internet]. Seattle, Wash: National Network of Libraries of Medicine, Pacific Northwest Region; Bethesda, MD: National Library of Medicine. 2006. [cited 2021 May 23]. Available from: http://resource.nlm.nih.gov/101276147

[22] Hamasu C, Kelly B. Assessment and evaluation is not a gut feeling: integrating assessment and evaluation into library operations. J Med Libr Assoc. 2013 Apr;101(2):85-7.

[23] Creamer A, Morales M, Crespo J, Kafel D, Martin ER. Assessment of health sciences and science and technology librarian e-science educational needs to develop an e-science web portal for librarians. J Med Libr Assoc. 2011 Apr;99(2):153-6.

[24] Creamer ME, Kafel D. Research data management and the health sciences librarian. In: Wood S, Editor. Health sciences librarianship. Chicago: Rowan \& Littlefield; 2014. p. 252-74. 